Supporting information

\title{
Highly Thermal Insulated and Super-Hydrophilic Corn Straw for Efficient Solar Vapor Generation
}

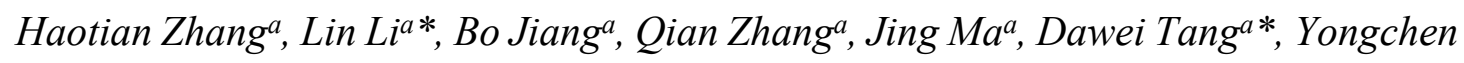
Song ${ }^{a}$

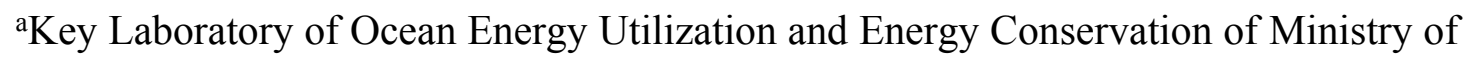
Education, School of Energy and Power Engineering, Dalian University of Technology, Dalian 116024, China

\section{*Corresponding auther}

E-mail: 1ilinnd@dlut.edu.cn (Lin Li); dwtang@dlut.edu.cn (Dawei Tang)

\section{Contents}

Note S1. Solar absorbance calculation method of flamed corn straw.

Note S2. Cost analysis of the double-layer flamed straw evaporator.

Figure S1. SEM images of the top surface of flamed straw.

Figure S2. Thermal conductivity of natural and flamed straw under different states.

Figure S3. Experimental equipment of solar vapor generation.

Figure S4. Mass change of water over time without optical illumination.

Figure S5. Scalable demonstration of the double-layer flamed straw evaporator.

Figure S6. The bilayer flamed straw can be fabricated with different thicknesses of the carbonized surface.

Table S1. Comparison of the material properties of different double-layer solar evaporators.

Table S2. Comparison of efficiency and cost of various solar vapor generation devices. 
Note S1. Solar absorbance calculation methods of flamed straw

Solar absorbance $(\alpha)$ is a measure of the ability of a material to absorb solar radiation and is broadly defined as a fraction between absorbed radiation and incoming solar radiation. Hence, solar absorbance can be calculated using the following equation:

$$
\alpha=\frac{\int_{\lambda_{\min }}^{\lambda_{\max }}[1-R(\lambda)] A(\lambda) d \lambda}{\int_{\lambda_{\min }}^{\lambda_{\max }} A(\lambda) d \lambda}
$$

where $\lambda$ is the wavelength, $A(\lambda)$ is the AM 1.5 solar spectral radiation defined by the ISO standard 9845-1(1992), $\lambda_{\min }$ and $\lambda_{\max }$ are, respectively, $300 \mathrm{~nm}$ and 2500 $\mathrm{nm}$, and $R(\lambda)$ is the total reflectance at the wavelength $\lambda$. 
Note S2. Cost analysis of the double-layer flamed straw evaporator.

The biomass-derived solar evaporator was designed with low system cost in mind. The design is entirely flamed corn straw and polymer foam based. The cost of each part is summarized using the price of similar items found on Alibaba. com as below:

- Flamed corn straw: corn straw could be obtained directly from the farmland without any cost because it's one of the agricultural residues with wide distribution, while the manufacturing price (surface carbonization) is estimated to be about $\$ 0.3$ $\mathrm{m}^{-2}$

- Polymer foam: $\$ 0.5 \mathrm{~m}^{-2}, 10 \mathrm{~mm}$ thick boards used.

Thus, the total cost of the flamed straw evaporator is around 0.8 dollars per square meter, and the cost is extraordinarily low. 




Figure S1. SEM images of the top surface of flamed straw. a) SEM image shows a large vascular bundle and the rough surface of natural straw after flaming treatment. b, c) Magnified SEM images of the rough surface, numerous carbon black nanoparticles are deposited on this surface, enhancing solar absorbance. 


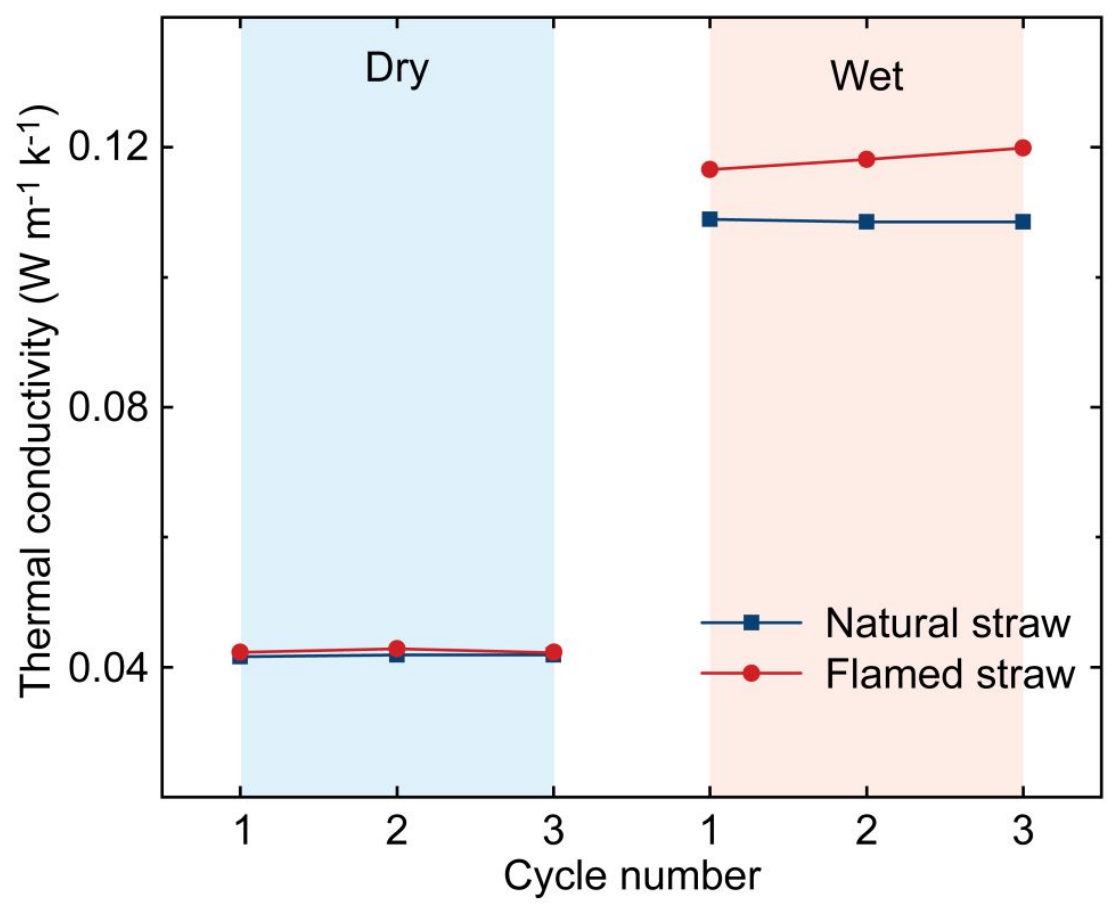

Figure S2. Thermal conductivity of natural and flamed straw under different states. 


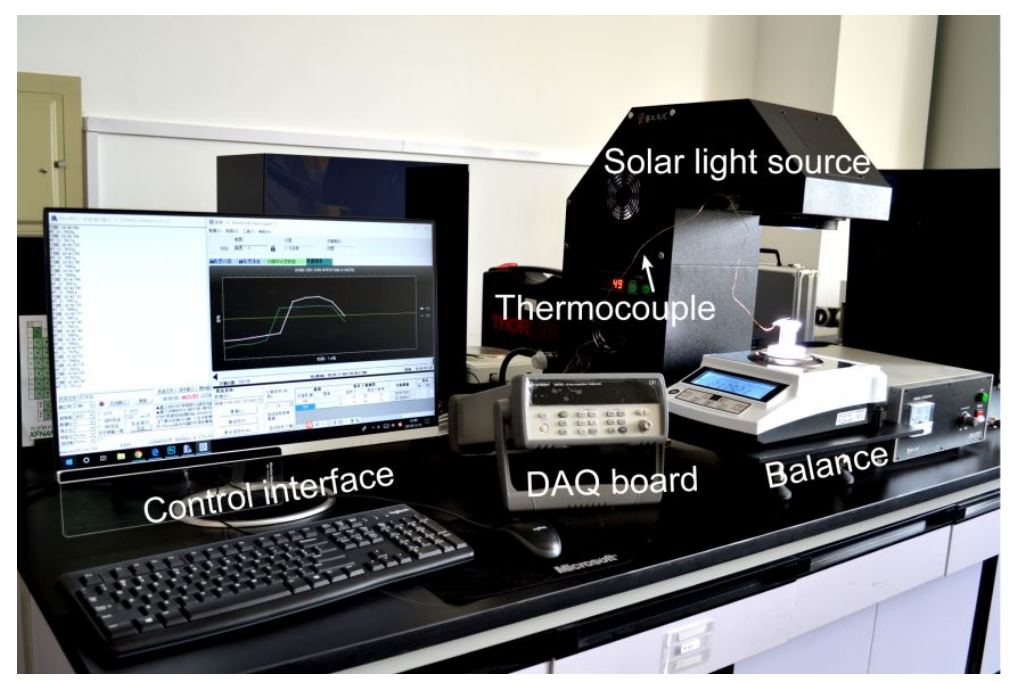

Figure S3. Experimental equipment of solar vapor generation. 


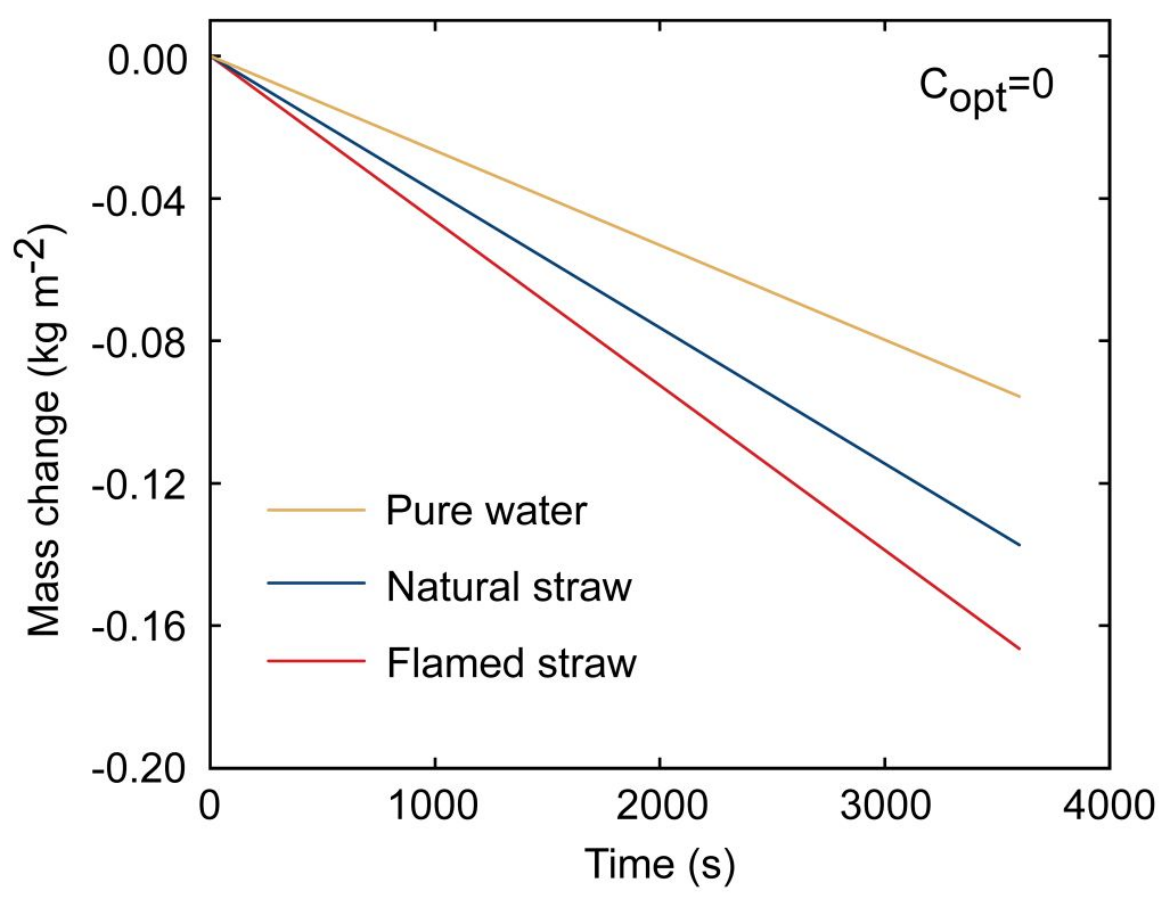

Figure S4. Mass change of water over time without optical illumination. 

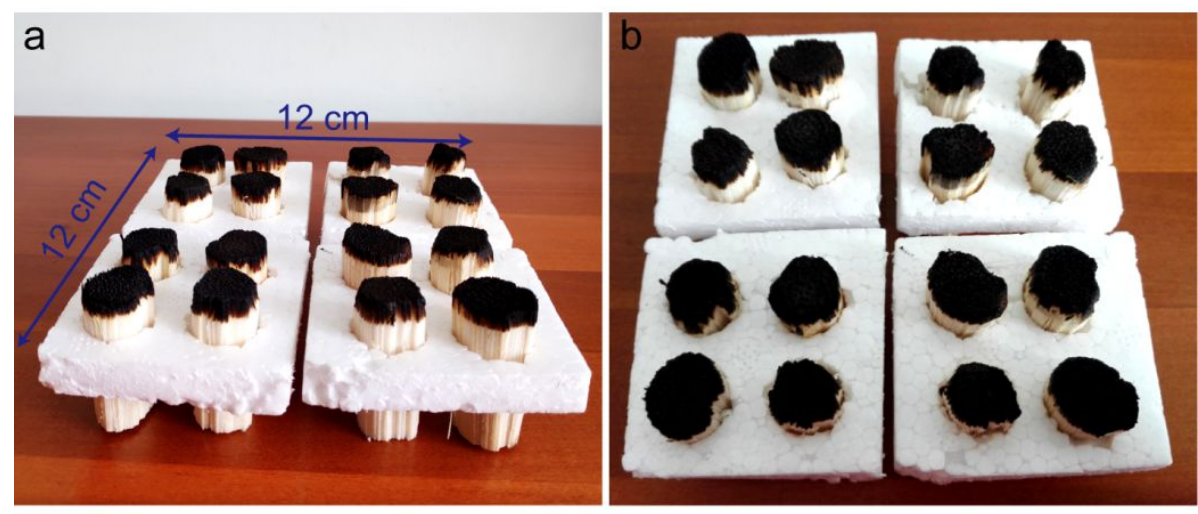

Figure S5. Scalable demonstration of the double-layer flamed straw evaporator. 


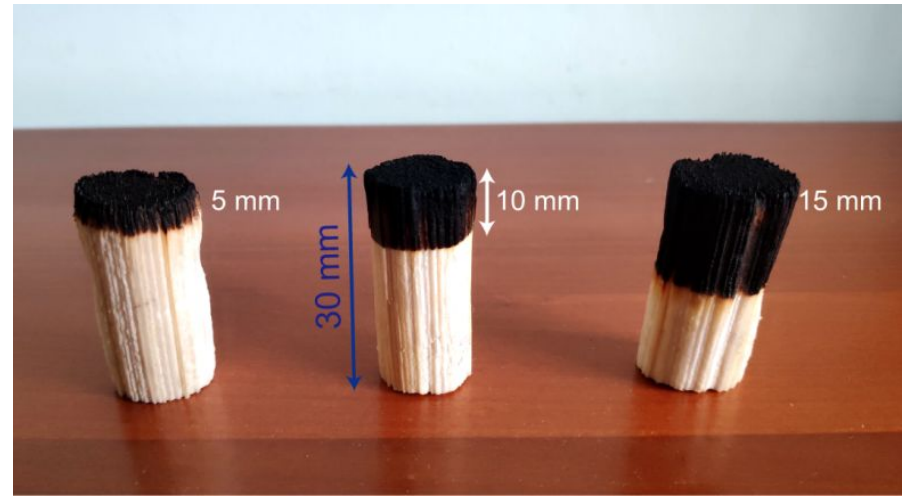

Figure S6. The bilayer flamed straw can be fabricated with different thicknesses of the carbonized surface. 
Table S1. Comparison of the material properties of different double-layer solar evaporators.

\begin{tabular}{|c|c|c|c|c|c|}
\hline \multirow{2}{*}{ Ref. } & \multirow{2}{*}{$\begin{array}{c}\text { Double-layer } \\
\text { evaporator } \\
\text { materials } \\
\text { (Upper / Bottom) }\end{array}$} & \multicolumn{2}{|c|}{ Hydrophilic / Hydrophobic } & \multirow{2}{*}{$\begin{array}{l}\text { Salt } \\
\text { rejectin } \\
\mathbf{g} \\
\text { propert } \\
\mathbf{y} \\
\end{array}$} & \multirow{2}{*}{$\begin{array}{l}\text { Energy } \\
\text { efficiency }\end{array}$} \\
\hline & & Upper layer & Bottom layer & & \\
\hline 1 & $\begin{array}{c}\text { CB-PMMA / PAN } \\
\text { (Thickness } 0.125 \\
\mathrm{~mm})\end{array}$ & Hydrophobic & Hydrophilic & $\sqrt{ }$ & $72 \%$ \\
\hline 2 & $\begin{array}{l}\text { HDMS-treated } \mathrm{SiO}_{2} \\
\text { / CNF-CNT aerogel } \\
\text { (Thickness 2 9 } \\
\text { mm) }\end{array}$ & Hydrophobic & Hydrophilic & $\sqrt{ }$ & $80 \%$ \\
\hline 3 & $\begin{array}{l}\text { Treated } \mathrm{Cu} \text { foam / } \\
\text { polystyrene foam } \\
\text { (Through holes) }\end{array}$ & Hydrophobic & Hydrophobic & $\sqrt{ }$ & $80 \%$ \\
\hline 4 & $\begin{array}{l}\text { CB-PAN / PVDF } \\
\text { (Through holes) }\end{array}$ & Hydrophilic & Hydrophobic & l & $82 \%$ \\
\hline 5 & $\begin{array}{c}\text { Carbonized poplar } \\
\text { wood }\end{array}$ & Hydrophilic & Hydrophilic & / & $86.7 \%$ \\
\hline 6 & $\begin{array}{c}\text { Surface } \\
\text { carbonization } \\
\text { sugarcane }\end{array}$ & Hydrophilic & Hydrophilic & l & $87.4 \%$ \\
\hline $\begin{array}{l}\text { This } \\
\text { work }\end{array}$ & Flamed corn straw & Hydrophilic & Hydrophilic & l & $86 \pm 2 \%$ \\
\hline
\end{tabular}


Table S2. Comparison of efficiency and cost of various solar vapor generation devices.

\begin{tabular}{|c|c|c|c|c|}
\hline Ref. & Materials & Cost & $\begin{array}{c}\text { Evaporation } \\
\text { rate } \\
\left(\mathrm{kg} \mathrm{m}^{-2} \mathbf{h}^{-1}\right) \\
\end{array}$ & $\begin{array}{c}\text { Efficiency } \\
(\%)\end{array}$ \\
\hline 7 & $\begin{array}{l}\text { Au/NPT plasmonic } \\
\text { absorbers }\end{array}$ & High & 1.0 & 63 \\
\hline 8 & $\begin{array}{l}\text { Nanoporous anodic } \\
\text { aluminium oxide } \\
\text { membrane (AAM) }\end{array}$ & Medium & 1.0 & 57 \\
\hline 9 & $\begin{array}{c}\text { Metasurface of } \\
\text { biomimetic nanoparticle }\end{array}$ & Medium & 1.18 & 76 \\
\hline 10 & $\begin{array}{l}\text { Graphite with carbon } \\
\text { foam }\end{array}$ & Medium & 1.2 & 64 \\
\hline 11 & $\begin{array}{l}\text { Nitrogen-doped porous } \\
\text { graphene }\end{array}$ & High & 1.5 & 80 \\
\hline 12 & $\begin{array}{c}\text { Graphene oxide-based } \\
\text { aerogel }\end{array}$ & High & 1.622 & 83 \\
\hline 13 & $3 \mathrm{D}$ printed $\mathrm{CNTs} / \mathrm{GO}$ & High & 1.355 & 85.6 \\
\hline 14 & CNTs/wood membrane & High & 0.95 & 65 \\
\hline 15 & Wood@POF & Medium & 1.95 & 79.6 \\
\hline 16 & $\begin{array}{l}\text { ALD/Chinese-ink-coated } \\
\text { wood }\end{array}$ & Medium & 1.31 & 82.2 \\
\hline 17 & Plasmonic wood & Medium & 1.1 & 67 \\
\hline 18 & Flame-treated wood & Low & 1.05 & 72 \\
\hline 19 & Carbonized mushroom & Low: $\$ 2 \mathrm{~kg}^{-1}$ & 1.475 & 78 \\
\hline 5 & Mesoporous wood & Low & 1.15 & 80 \\
\hline 6 & $\begin{array}{l}\text { Surface-carbonized } \\
\text { sugarcane }\end{array}$ & Low & 1.57 & 87.4 \\
\hline 20 & Carbonized bamboos & Low & 1.191 & 84 \\
\hline $\begin{array}{l}\text { This } \\
\text { work }\end{array}$ & Flamed corn straw & Low: $\$ 0.8 \mathrm{~m}^{-2}$ & 1.497 & $86 \pm 2$ \\
\hline
\end{tabular}

Notes: The energy efficiency are calculated data under one sun illumination. 


\section{References}

(1) Xu, W.; Hu, X.; Zhuang, S.; Wang, Y.; Li, X.; Zhou, L.; Zhu, S.; Zhu, J. Flexible and Salt Resistant Janus Absorbers by Electrospinning for Stable and Efficient Solar Desalination. Adv. Energy Mater. 2018, 8, 1702884.

(2) Hu, R.; Zhang, J.; Kuang, Y.; Wang, K.; Cai, X.; Fang, Z.; Huang, W.; Chen, G.; Wang, Z. A Janus evaporator with low tortuosity for long-term solar desalination. $J$. Mater. Chem. A 2019, 7, 15333-15340.

(3) Xu, N.; Li, J.; Wang, Y.; Fang, C.; Li, X.; Wang, Y.; Zhou, L.; Zhu, B.; Wu, Z.; Zhu, S.; Zhu, J. A water lily-inspired hierarchical design for stable and efficient solar evaporation of high-salinity brine. Sci. $A d v$. 2019, 5, eaaw7013.

(4) Gao, T.; Li, Y.; Chen, C.; Yang, Z.; Kuang, Y.; Jia, C.; Song, J.; Hitz, E. M.; Liu, B.; Huang, H.; Yu, J.; Yang, B.; Hu, L. Architecting a Floatable, Durable, and Scalable Steam Generator: Hydrophobic/Hydrophilic Bifunctional Structure for Solar Evaporation Enhancement. Small Methods 2019, 3, 1800176.

(5) Jia, C.; Li, Y.; Yang, Z.; Chen, G.; Yao, Y.; Jiang, F.; Kuang, Y.; Pastel, G.; Xie, H.; Yang, B.; Das, S.; Hu, L. Rich Mesostructures Derived from Natural Woods for Solar Steam Generation. Joule 2017, 1, 588-599.

(6) Liu, J.; Liu, Q.; Ma, D.; Yuan, Y.; Yao, J.; Zhang, W.; Su, H.; Su, Y.; Gu, J.; Zhang, D. Simultaneously achieving thermal insulation and rapid water transport in sugarcane stems for efficient solar steam generation. J. Mater. Chem. A 2019, 7, 90349039.

(7) Zhou, L.; Tan, Y.; Ji, D.; Zhu, B.; Zhang, P.; Xu, J.; Gan, Q.; Yu, Z.; Zhu, J. Selfassembly of highly efficient, broadband plasmonic absorbers for solar steam generation. Sci. Adv. 2016, 2, e1501227.

(8) Zhou, L.; Tan, Y.; Wang, J.; Xu, W.; Yuan, Y.; Cai, W.; Zhu, S.; Zhu, J. 3D selfassembly of aluminium nanoparticles for plasmon-enhanced solar desalination. Nat. Photonics 2016, 10, 393-398.

(9) Liu, C.; Huang, J.; Hsiung, C.-E.; Tian, Y.; Wang, J.; Han, Y.; Fratalocchi, A. High-Performance Large-Scale Solar Steam Generation with Nanolayers of Reusable Biomimetic Nanoparticles. Adv. Sustainable Syst. 2017, 1, 1600013.

(10) Ghasemi, H.; Ni, G.; Marconnet, A. M.; Loomis, J.; Yerci, S.; Miljkovic, N.; Chen, G. Solar steam generation by heat localization. Nat. Commun. 2014, 5, 4449.

(11) Ito, Y.; Tanabe, Y.; Han, J.; Fujita, T.; Tanigaki, K.; Chen, M. Multifunctional Porous Graphene for High-Efficiency Steam Generation by Heat Localization. $A d v$. Mater. 2015, 27, 4302-4307.

(12) Li, X.; Xu, W.; Tang, M.; Zhou, L.; Zhu, B.; Zhu, S.; Zhu, J. Graphene oxidebased efficient and scalable solar desalination under one sun with a confined 2D water path. P. Natl. Acad. Sci. USA 2016, 113, 13953-13958.

(13) Li, Y.; Gao, T.; Yang, Z.; Chen, C.; Luo, W.; Song, J.; Hitz, E.; Jia, C.; Zhou, Y.; Liu, B.; Yang, B.; Hu, L. 3D-Printed, All-in-One Evaporator for High-Efficiency Solar Steam Generation under 1 Sun Illumination. Adv. Mater. 2017, 29, 1700981. 
(14) Chen, C.; Li, Y.; Song, J.; Yang, Z.; Kuang, Y.; Hitz, E.; Jia, C.; Gong, A.; Jiang, F.; Zhu, J. Y.; Yang, B.; Xie, J.; Hu, L. Highly flexible and efficient solar steam generation device. Adv. Mater. 2017, 29, 1701756.

(15) Xia, Z.-J.; Yang, H.-C.; Chen, Z.; Waldman, R. Z.; Zhao, Y.; Zhang, C.; Patel, S. N.; Darling, S. B. Porphyrin Covalent Organic Framework (POF)-Based Interface Engineering for Solar Steam Generation. Adv. Mater. Interfaces 2019, 6, 1900254.

(16) Yang, H.-C.; Chen, Z.; Xie, Y.; Wang, J.; Elam, J. W.; Li, W.; Darling, S. B. Chinese Ink: A Powerful Photothermal Material for Solar Steam Generation. $A d v$. Mater. Interfaces 2019, 6, 1801252.

(17) Zhu, M.; Li, Y.; Chen, F.; Zhu, X.; Dai, J.; Li, Y.; Yang, Z.; Yan, X.; Song, J.; Wang, Y.; Hitz, E.; Luo, W.; Lu, M.; Yang, B.; Hu, L. Plasmonic wood for highefficiency solar steam generation. Adv. Energy Mater. 2018, 8, 1701028.

(18) Xue, G.; Liu, K.; Chen, Q.; Yang, P.; Li, J.; Ding, T.; Duan, J.; Qi, B.; Zhou, J. Robust and low-cost flame-treated wood for high-performance solar steam generation. ACS Appl. Mater. Interfaces 2017, 9, 15052-15057.

(19) Xu, N.; Hu, X.; Xu, W.; Li, X.; Zhou, L.; Zhu, S.; Zhu, J. Mushrooms as efficient solar steam-generation devices. Adv. Mater. 2017, 29, 1606762.

(20) Li, Z.; Wang, C.; Lei, T.; Ma, H.; Su, J.; Ling, S.; Wang, W. Arched bamboo charcoal as interfacial solar steam generation integrative device with enhanced water purification capacity. Adv. Sustainable Syst. 2019, 3, 1800144. 